\title{
Renal Outcome after Combined Liver-kidney Transplantation in Children
}

Laura Burgos $^{1 *}$, Maria Jose Martinez Urrutia1 ${ }^{1}$, Francisco Hernandez ${ }^{3}$, Laura Espinosa ${ }^{2}$, Roberto Lobato', Pedro Lopez ${ }^{1}$, Manolo Lopez Santamaria ${ }^{3}$ and Enrique Jaureguizar ${ }^{1}$

${ }^{1}$ Paediatric Urology, University Hospital La Paz, Madrid, Spain

${ }^{2}$ Paediatric Nephrology, University Hospital La Paz, Madrid, Spain

${ }^{3}$ Paediatric Surgery, University Hospital La Paz, Madrid, Spain

\begin{abstract}
Background: Combined Liver and Kidney Transplantation (CLKT) is a recognized treatment option for a select group of paediatric patients with severe liver and kidney disease. The aim of this study is to report Indication and outcome of CLKT in our center and to compare the results with the Isolated Kidney Transplantation (IKT).
\end{abstract}

Patients and method: We retrospectively reviewed children who underwent CLKT between 1997 and 2009. Data analyzed include age, sex, cause of kidney/liver disease, pre-transplant dialysis, donor age, cross-match, and immunosuppression regimen. Additionally, cold ischemia time, Acute Rejection (AR) episodes and patient and graft survival were compared with patients who had IKT in the same period of time.

Results: 9 children (5 females, 4 males) underwent CLKT and 127 IKT. In CLKT group, mean patient age was $10.5 \pm 3.9$ years. Indications for CLKT were: primary hyperoxaluria $(n=3)$, hemolytic uremic syndrome $(n=1)$, Alagille Syndrome $(n=1)$, nephronoptisis $(n=1)$ and polycystic hepatorenal disease $(n=3)$. Five patients were on dialysis before transplant. In all patients, both grafts were obtained from the same donor and cross-matches were negative. Immunosuppression was induced with Basiliximab and maintained with triple therapy (Tacrolimus+Mycophenolate mofetil+Prednisone). Mean cold ischemia time was shorter in CLKT (10.9 \pm 3.5 vs. $16.8 \pm 4.6$ hours) and hospital stay was longer ( $35.5 \pm 10$ vs. $13.9 \pm 5.3$ days) compared to IKT. Long-term mean Glomerular Filtration Rate (GFR) and cystatin levels were similar in both groups. Rejection tended to be less frequent in CLKT (11 vs. $25 \%)$ than in IKT. Patient survival was lower $(P=0.008)$ in CLKT. One patient with chronic liver and kidney rejection due to noncompliance died during re-transplantation 5 years after the first transplant. There were no deaths in the IKT group Mean follow-up time is $5.27 \pm 2.9$ years the IKT group Mean Follow-up time is $5.27 \pm 2.9$ years.

Conclusions: Most common indications for CLKT in our children are primary hyperoxaluria and polycystic disease. Long-term results in children receiving CLKT are comparable to those with isolated kidney transplantation.

Keywords: Combined transplantation; Hyperoxaluria; Liver transplantation; Kidney transplantation

\section{Introduction}

Combined liver and kidney transplantation is a treatment option for End-Stage Renal Disease (ESRD) with accompanying liver disease or isolated enzymatic deficiencies of the liver. Most common indication for CLKT is Primary Hyperoxaluria (PH-1), which is a hepatic deficiency of the liver enzyme Alanine Glycoxylate Transaminase (AGT), with autosomal recessive inheritance that leads to overproduction of oxalate that must be excreted by kidneys [1]. Other than that, frequent indications for CLKT are: polycystic hepatorenal disease, atypical haemolytic uremic syndrome, congenital hepatic fibrosis and other metabolic disorders affecting the kidney, where the liver is transplanted to correct the enzymatic deficiency, like alpha- 1 antitrypsin deficiency or methylmalonic acidemia [1].

Since 1997, CLKT has been our choice treatment in children with ESRD associated to hepatic failure. It still remains controversy regarding the right timing for this procedure. Our aim with this study is to report our experience with this treatment for different indications and its outcome in our center. Finally, a reference to Isolated Cadaveric Renal Transplantation (IKT), compared to CLKT, is shown.

\section{Patients and Method}

Between 1997 and 2009, 9 patients in our institution underwent simultaneous CLKT. We performed a retrospective study of medical charts, collecting data about: sex and age at transplant, cause of liver and kidney disease, need of pretransplant dyalisis or previous transplants and patient medical situation at transplant. We also gathered information about donor age and weight, cross-match, cold ischemia time, immunosuppression regimen and technical transplant details. Finally postoperative outcome was studied analysing data about: Acute Rejection (AR) episodes, hospital stay and patient and graft survival. Mean Glomerular Filtration Rate (GFR) was calculated using modified Schwartz formula [2].

Cadaveric renal transplants performed in the same period of time were reviewed collecting information about cold ischemia time, hospital stay, AR episodes and graft function; these variables were compared to patients in the group of CLKT. T test was used to compare quantitative variables and Chi2 test for qualitative variables, log Rank test was used to compare survival between groups.

In our unit, children with $\mathrm{PH}-1$ do not undergo preoperative renal support routinely, except those patients who were already in dialysis, in order to decrease blood oxalic acid levels prior to transplantation. In the postoperative period, they do not undergo dialysis either but they receive intensive medical treatment to decrease stone formation. Considering urine levels of oxalic acid may take very long to decrease,

*Corresponding author: Dra Laura Burgos Lucena, Urologia Pediatrica Hospital Universitario La Paz, Madrid, Spain, Tel: +34-639227406; E-mail: lauraburgos33@hotmail.com

Received November 26, 2013; Accepted December 17, 2013; Published December 18, 2013

Citation: Burgos L, Martinez Urrutia MJ, Hernandez F, Espinosa L, Lobato R, et al (2013) Renal Outcome after Combined Liver-kidney Transplantation in Children. J Transplant Technol Res 4: 125. doi:10.4172/2161-0991.1000125

Copyright: $\odot 2013$ Burgos L, et al. This is an open-access article distributed under the terms of the Creative Commons Attribution License, which permits unrestricted use, distribution, and reproduction in any medium, provided the original author and source are credited. 
post-transplant treatment includes aggressive fluid management and oral intake of citrate.

In all patients, both grafts were obtained from the same donor. Cross-matches were negative and they all received organs from compatible blood group cadaveric donors. The whole liver and a solitary kidney were used in all cases. The transplantation procedure always followed the standard technique, placing the liver graft before the kidney implantation. Kidney was always placed in extraperitoneal position, in the right iliac fossa and through the original extended incision.

\section{Results}

Between 1997 and 2011, 9 children underwent CLKT (5 females and 4 males) with a mean age at transplant of 10.53 .9 years. Most frequent indications in our series were primary hyperoxaluria $(n=3)$ and polycystic hepatorenal disease $(n=3)$, followed by atypical haemolytic uremic syndrome $(\mathrm{n}=1)$, Alagille syndrome $(\mathrm{n}=1)$ and nephronoptisis $(n=1)$. Diagnosis was confirmed by the progression to ESRD, renal biopsy and genetic study. Mean donor age was $8.5 \pm 3.5$ years. Our patients' pretransplant characteristics are summarized in Table 1.

Combined pre-emptive transplantation was possible in 4 patients. The remaining 5 patients needed dialysis before transplant, that was always hemodialysis, with a mean time of pretransplant duration of $22.4 \pm 29$ months. One patient had a previous renal transplantation 9 years before the combined transplant due to a nephronoptisis that led her to renal failure before liver disease.

Immunosuppression was induced with Basiliximab and maintained with triple therapy (Tacrolimus+Mycophenolate mofetil+Prednisone) in all patients. Along the follow-up biopsies were taken when indicated by clinical or biochemical values. Patients' postoperative situation is summarized in Table 2 .

In the same period of time, 127 IKT from cadaveric donors were performed. Data of CLKT and KT were compared (Table 3): mean cold ischemia time for renal implantation was lower in CLKT compared to KT (10.9 \pm 3.5 vs. $16.8 \pm 4.6$ hours) while mean duration of hospital stay was longer ( $35.5 \pm 10$ vs. $13.9 \pm 5.3$ days). Acute rejection episodes tended to be less frequent, although not significant, in CLKT compared to KT ( $11 \%$ vs. $25 \%)$. The only kidney Acute Rejection (AR) episode in the CLKT group occurred one month after transplantation and responded to corticosteroid therapy with good posterior outcome. Patient survival was lower (Log Rank test; $\mathrm{P}=0.008$ ) in CLKT, only one patient died, he suffered from chronic liver and kidney rejection due to non-compliance and died during retransplantation 5 years after the first transplant. There were no deaths in the IKT group, but 24 grafts were lost. Twelve of the graft loss was due to chronic rejection (confirmed by biopsy) and 2 due to renal artery thrombosis. No graft was lost due to acute rejection. After a mean follow-up period of $5.3 \pm 3$ years, mean GFR is similar in CLKT and KT ( $97.5 \pm 19.9$ vs. $97 \pm 33 \mathrm{ml} /$ $\mathrm{min} / 1.73 \mathrm{~m}^{2}$ ) and mean cystatin level was also similar in both groups $1.2 \pm 0.28 \mathrm{mg} / \mathrm{l}$ vs. $1.3 \pm 0.6$.

In the CLKT group, all grafts started functioning in the immediate postoperative period except in one patient (number 9), with a delay of one hour after reperfusion. One patient (number 9) needed liver retransplantation due to hepatic artery thrombosis with no effect on renal function. Donor weight and age were $35 \mathrm{~kg}$ and 10 years old while recipient weight and age were $33 \mathrm{~kg}$ and 11 years old. No patient underwent postoperative dialysis.

Among the 3 patients with hyperoxaluria, only one of them was on pre-trasplant dialysis, but he did not have systemic deposits. After transplantation, oxalic urinary levels were measured periodically in these 3 patients and became normal after a mean time of $18 \pm 6$ months. One patient with chronic liver and kidney rejection due to treatment non-compliance died during retransplantation 5 years after the first transplant.

According to the organ allocation system in our country, patients waiting for combined liver and kidney do not have priority compared to isolate liver. Patients received both grafts attending just the criteria

\begin{tabular}{|c|c|c|c|c|c|c|}
\hline Patients & Sex & Age (years) & Indications & PREtranspl dialysis & Time in dialysis (months) & PREtranspl situation (GFR) \\
\hline $1 \mathrm{RBL}$ & $\mathrm{M}$ & 9.5 & Polycystic sd & Yes & 9 & ESRD \\
\hline $2 \mathrm{DBC}$ & M & 9 & HUS & Yes & 74 & ESRD \\
\hline $3 \mathrm{PCL}$ & $\mathrm{F}$ & 29 & Alagille & No & No & 34 \\
\hline 4 GGR & M & 7,4 & Hyperoxaluria & Yes & 13 & ESRD \\
\hline $5 \mathrm{AGL}$ & $\mathrm{F}$ & 15 & Hyperoxaluria & No & No & 24 \\
\hline $6 \mathrm{AMC}$ & $\mathrm{F}$ & 10 & Polycystic sd & Yes & 12 & ESRD \\
\hline $7 \mathrm{NRM}$ & $\mathrm{F}$ & 14,5 & Polycysctic sd & No & No & 23 \\
\hline $8 \mathrm{ASM}$ & $\mathrm{F}$ & 14 & Nephronoptisis & Yes & 4 & ESRD \\
\hline 9 GVG & $M$ & 11,5 & Hyperoxaluria & No & No & 43,1 \\
\hline
\end{tabular}

Table 1: Patients characteristics before CLKT.

\begin{tabular}{|c|c|c|c|c|c|}
\hline Patients & Donor age (y.o) & Cold ischemia time (hr) & Follow-up (years) & POST situation (GFR in $\mathrm{ml} / \mathrm{min} / 1.73 \mathrm{~m}^{2}$ ) & Hospital stay (days) \\
\hline $1 \mathrm{RBL}$ & 11,5 & 11,0 & 8,99 & - & - \\
\hline $2 \mathrm{DBC}$ & 3,8 & 10,45 & 1,77 & 110,0 & 36 \\
\hline $3 \mathrm{PCL}$ & 4 & 10,41 & 9,59 & 93,40 & 46 \\
\hline 4 GGR & 12 & 9,0 & 7,37 & 125,10 & 29 \\
\hline $5 \mathrm{AGL}$ & 8 & 18,0 & 5,43 & - & - \\
\hline $6 \mathrm{AMC}$ & 7 & 9,0 & 3,03 & 116,7 & 27 \\
\hline $7 \mathrm{NRM}$ & 14 & 11,0 & 4,56 & 74,10 & 34 \\
\hline 8 ASM & 7 & 6,3 & 6,88 & 77,0 & 25 \\
\hline 9 GVG & 10 & 13,75 & 0,36 & 86,4 & 52 \\
\hline MEAN & $8,5 \pm 3,5$ & $10,9 \pm 3,5$ & $5.3 \pm 3$ & $97.5 \pm 19$ & $35.5 \pm 10$ \\
\hline
\end{tabular}

Table 2: Patient's postoperative situation. 
for liver transplant. The group can internally decide to priorize patients waiting for CLKT, but because of the dramatic shortage of pediatric donors, this policy barely has any influence in the probability of transplant.

Data about receptor and donor weight are resumed in Table 4.

\section{Discussion}

As in previous publications $[1,3]$, primary hiperoxaluria $(\mathrm{PH}-1)$ was one of the main indication for CLKT in our center. This enzymatic deficiency leads to overproduction and excretion of oxalate causing progressive renal failure by the age of 15 [3]. Patients with End Stage Renal Disease (ESRD) have higher serum level of oxalate that is deposited in tissues like retina and myocardium. It has been published that systemic deposition of oxalate crystals begins when GFR falls bellow $40 \mathrm{ml} / \mathrm{min} / 1.73 \mathrm{~m}^{2}$ [4]. Since these patients are usually diagnosed once renal failure develops, a combined transplant becomes necessary.

In this group of patients, it is not clear yet how to manage the large oxalate load after transplant. Some authors [5] recommend postoperative dialysis to prevent nephrocalcinosis in patients with important systemic involvement, acute tubular necrosis or delayed graft function although the effect on the long-term renal function has not been documented yet. Considering that none of our patients had tubular necrosis, that all the grafts started working in the immediate postoperative period and that once the graft is working properly, diuresis should be enough to filter blood oxalic acid naturally, in our experience, postoperative dialysis is not necessary. In our unit $\mathrm{PH}-1$ patients are managed with intensive medical treatment to decrease stone formation, consisting in high fluid intake and the use of crystallization inhibitors.

It has been published that children with PH-I seem to have delayed recovery of renal function after CLKT compared to those with polycystic disease $[4,6]$. Considering that in our series, diuresis started immediately after transplant, post-transplant dialysis was not necessary, hospital stay was the same as in any other patient and final GFR was similar to other groups, in our experience, postoperative evolution was similar despite the underlying disease etiology.

\begin{tabular}{|c|c|c|}
\hline & Receptor weight $\mathbf{( k g )}$ & Donor weight \\
\hline Patient 1 & 25 & 27.30 \\
\hline Patient 2 & 32.10 & 32.13 \\
\hline Patient 3 & 7.50 & 18 \\
\hline Patient 4 & 30 & 30 \\
\hline Patient 5 & 27 & 30 \\
\hline Patient 6 & 52.90 & 55 \\
\hline Patient 7 & 26.50 & 28 \\
\hline Patient 8 & 22.40 & 15 \\
\hline Patient 9 & 33.30 & 35 \\
\hline Mean & $28.5 \pm 5.12$ & $30.14 \pm 4.93$ \\
\hline & Table 3: Donor and receptor weight. \\
\hline
\end{tabular}

\begin{tabular}{|l|c|c|}
\hline \multicolumn{1}{|c|}{} & CLKT & IKT \\
\hline Ischemia time $(\mathrm{hr})$ & $10.99 \pm 3.3$ & $16.8 \pm 4.6$ \\
\hline Hospital stay (days) & $35.57 \pm 10$ & 13.95 .3 \\
\hline Acute rejection episodes & $11 \%$ & $25 \%$ \\
\hline Long-germ GFT $\left(\mathrm{ml} / \mathrm{min} / 1.73 \mathrm{~m}^{2}\right)$ & $97.5 \pm 19$ & $97 \pm 33$ \\
\hline Cystatin $(\mathrm{mg} / \mathrm{l})$ & $1.2 \pm 0.28$ & $1.3 \pm 0.6$ \\
\hline Total $(1997-2009)$ & 9 & 127 \\
\hline
\end{tabular}

Table 4: CLKT compared to IKT.
Autosomal recessive polycystic kidney disease is an inherited condition that leads to ESRD early in life. Liver involvement includes Caroli disease and congenital hepatic fibrosis. Despite the limited published data, CLKT is accepted as the choice treatment in polycystic disease with end-stage organ failure as it provides an immunologic advantage and reduces rejection rates [4]. In our series it was the second cause for CLKT with good results in terms of hepatic and renal function.

Atypical hemolytic uremic syndrome is due to factor $\mathrm{H}$ deficiency which is produced by the liver and has a main role in the regulation of alternate complement activation pathway. Clinically it presents end-stage renal failure, microangiopathic haemolytic anemia, thrombocytopenia and hypertension. Our only patient with this syndrome had a factor B deficiency that was provided by the liver graft at the time of transplantation (since Eculizumab was not available by that time); after monitoring it, complement became normal. Outcome after CLKT is variable [7,8], but in our short experience, patient showed normal values of GFR and cystatin levels after 3 years of follow-up.

Congenital hepatic fibrosis is characterized by fibrous enlargement of portal tracts that contain abnormal bile ducts and it is quite often associated with renal disease [9]. Hepatosplenomegaly and portal hypertension make porto-systemic shunt necessary. None of our patients suffered that condition (except in association with polycystic renal disease) but, in those cases were shunt procedures fail, it has been reported that CLKT may have good results [10].

There is still controversy regarding the best timing for CLKT. In our experience, ideally it should be performed before the patient needs to enter dialysis. Our unit's criteria to start dialysis are a GFR $\leq 10 \mathrm{ml} / \mathrm{min} / 1.73 \mathrm{~m}^{2}$ or impossibility to clinically manage the patient with conservative treatment. Even though pre-emptive transplantation was not always possible (only in 4 out of our 9 patients), we agree with literature that extended dialysis and poor general condition negatively affect recovery and survival after transplant (3).

All our patients received organs from blood group compatible cadaveric donors. It has been previously proven that CLKT can protect the kidney against hyperacute rejection in positive cross-match patients [11]. In our series pretransplant lymphocyte cross-matches were negative in all patients, probably because it was the first transplant, but we do not consider it strictly necessary.

In the same period of time, 127 IKT from cadaveric donors were performed. Even though the great difference in the number of patients makes results difficult to compare, we observed that cold ischemia time is much shorter in CLKT since the liver needs a shorter ischemia time. On the other hand, hospital stay is much longer in CLKT compared to IKT because these patients have a greater need of intensive medical treatment. However, acute rejection episodes tended to be less frequent in CLKT, supporting that liver transplantation is immunologically protective of the kidney allograft as it has been proven previously (6), although the disparity of number between both groups limits the value of statistical analysis. Single center reports have been more promising, reporting acute renal allograft rejection rates of $4.2 \%$ in CLKT compared to $32.5 \%$ in well-matched kidney transplants [12]. On the other hand, GFR and cystatin levels are quite similar in both groups at the end of follow-up (Table 3).

\section{Conclusion}

Most common indications for CLKT in our children are primary hyperoxaluria and polycystic disease. Even though complexity of 
Citation: Burgos L, Martinez Urrutia MJ, Hernandez F, Espinosa L, Lobato R, et al. (2013) Renal Outcome after Combined Liver-kidney Transplantation in Children. J Transplant Technol Res 4: 125. doi:10.4172/2161-0991.1000125

CLKT is higher long-term results in these children are comparable to those with IKT.

\section{References}

1. Sutherland SM, Alexander SR, Sarwal MM, Berquist WE, Concepcion W (2008) Combined liver-kidney transplantation in children: indications and outcome. Pediatr Transplant 12: 835-846.

2. Tsampalieros A, Lepage N, Feber J (2011) Intra-individual variability of the modified Schwartz and novel CKiD GFR equations in pediatric renal transplant patients. Pediatr Transplant 15: 760-765.

3. Chava SP, Singh B, Pal S, Dhawan A, Heaton ND (2009) Indications for combined liver and kidney transplantation in children. Pediatr Transplant 13: 661-669

4. Perera MT, McKiernan PJ, Sharif K, Milford DV, Lloyd C, et al. (2009) Renal function recovery in children undergoing combined liver kidney transplants. Transplantation 87: 1584-1589.

5. Cochat P, Liutkus A, Fargue S, Basmaison O, Ranchin B, et al. (2006) Primary hyperoxaluria type I: still challenging! Pediatr Nephrol 21: 1075-1081.
6. De la Cerda F, Jimenez WA, Gjertson DW, Venick R, Tsai E, et al. (2010) Rena graft outcome after combined liver and kidney transplantation in children: UCLA and UNOS experience. Pediatr Transplant 14: 459-64.

7. Remuzzi G, Ruggenenti P, Codazzi D (2002) Combined kidney and liver tranplantation for familial haemolytic uraemic syndrome. Lancet 359: 1671 1672.

8. Saland JM, Emre SH, Shneider BL (2006) Favorable long-term outcome after liver-kidney transplant for recurrent hemolytic uremic syndrome associated with a factor H mutation. Am J Transplant 6: 1948-1952.

9. Di Bisceglie AM, Befeler AS, Schiff ER, Sorrell MF, Maddrey WC (2003) Nodular and cystic diseases of the liver. Diseases of the Liver. (9thedn), Lippincott Willians and Wilkins, Philadelphia, PA, USA, 1149-1168.

10. Grewal H, Brady L, Cronin DC 2nd (2000) Combined liver and kidney transplantation in children. Transplantation 70: 100-105.

11. Olausson M, Mjornstedt L, Norden G (2007) Successful combined partial auxiliary liver and kidney transplantation in highly sensitized cross-match positive recipients. Am J Transplant 7: 130-136.

12. Creput C, Durrbach A, Samuel D (2003) Incidence of renal and liver rejection and patient survival rate following combined liver and kidney transplantation. Am J Transplant 3: 348-356. 